\title{
Information Theory based Performance Analysis and Enhancement of Safety Applications in DSRC
}

\author{
Zhongyi Shen, Xin Zhang, Zhihao Chen, Meng Zhang, Yunlong Gu and Jinxi Fu \\ School of Information and Communication Engineering \\ Beijing University of Posts and Telecommunications \\ PO Box93, 10 XiTuCheng Rd, HaiDian, Beijing, CHINA, 100876 \\ Email: szy823804813@bupt.edu.cn
}

\begin{abstract}
Dedicated short-range communication (DSRC) is an enabling technology for Intelligent Transportation Systems (ITS). Due to lack of stable topology, the periodic and eventdriven safety applications take broadcast as an effective approach to disseminate messages, which leads to significant performance degradation in high density and highly dynamic scenarios. This paper presents an information theory based model to determine the theoretical amount of information required by DSRC safety applications, and the results are helpful in reducing redundant information in performance enhancement as well as gaining more insight for system design. Based on the model presented, an adaptive algorithm is proposed to derive the optimal safety message rate in different scenarios to reduce the channel load. Numerical simulations based on NS-3 show the proposed algorithm can improve the performance dramatically, and the effectiveness of the safety applications is guaranteed.
\end{abstract}

1

Keywords-Dedicated short-range communication (DSRC), safety message broadcast, information theory,mobility, entropy

\section{INTRODUCTION}

Dedicated Short-Range Communications (DSRC) is a wireless technology for intelligent transportation systems (ITS) to provide safety applications and to improve traffic efficiency for vehicular transportation. Growing attention from researchers and transportation industry has been driven by DSRC technology which employs IEEE 802.11p and IEEE 1609 standards for its capability to improve the traffic safety via various safety applications, through vehicle-to-vehicle $(\mathrm{V} 2 \mathrm{~V})$ and vehicle-toinfrastructure (V2I) communications.

The core of the DSRC-based applications are safety applications, including periodic broadcast and emergency message dissemination which are used for cooperative collision avoidance (CCA), and it's the primary motivation for deploying DSRC. Periodic broadcast messages containing safety information such as position, velocity etc. are disseminated among vehicles with DSRC-based equipment, and the message received form DSRC-equipped neighbors can be used to locate the vehicle in a collision threat or in blind spot. An emergency message will be send when accidents like collision or a sudden brake are detected. The U.S. Department of Transportation

\footnotetext{
${ }^{1}$ The research is supported by National Science and Technology Major Project of the Ministry of Science and Technology and project name is Wireless mobile spectrum research and verification for WRC15 with 2014ZX03003013-004 and the Fundamental Research Funds for the Central Universities under grant No.2014ZD03-02.
}

(DOT) has estimated that vehicle-to-vehicle (V2V) communication based on DSRC can address up to $82 \%$ of all crashes in the United States involving unimpaired drivers, potentially saving thousands of lives and billions of dollars [1].

The U.S. Federal Communications Commission allocated $75 \mathrm{MHz}$ of licensed spectrum in the $5.9 \mathrm{GHz}$ band for DSRC [2], and the spectrum is divided in to seven $10-\mathrm{MHz}$ channels, including a control channel $(\mathrm{CCH})$ for control message and safety related applications, and six service channels $(\mathrm{SCH})$ which will be used for non-safety applications. In order to ensure that all the control messages and safety messages won't be missed, DSRC devices will follow a synchronization procedure. A synchronization interval comprises a $\mathrm{CCH}$ interval, followed by a $\mathrm{SCH}$ interval. During the $\mathrm{CCH}$ interval, devices will tune to the $\mathrm{CCH}$ for safety applications and service announcement, and the SCH offered will be indicated. To handle time-critical safety applications, the broadcast mode is considered to be a highly appropriate technique for safety messages dissemination [3]. In this paper, safety-related information will be broadcast at a certain frequency, and a broadcast-based retransmission mechanism [4] is introduced to improve reliable of event-driven emergency message dissemination.

The main challenges of DSRC are packets collision and medium access congestion in medium access control (MAC) layer of IEEE $802.11 \mathrm{p}$ in dense and high mobility situation. At high density scenario, there are a large amount of devices in the transmission range of each vehicle, which will leads to significant performance degradation of IEEE 802.11p Carrier Sense Multiple Access (CSMA) and serious channel congestion [5]. The latency and low reliability resulting from the channel congestion and packets collision will impact the broadcastbased safety applications. Due to lack of request-to-send/clearto-send (RTS/CTS) process in IEEE $802.11 \mathrm{p}$ broadcast mode, the reliability of the transmission of event-driven emergency messages will decrease.

The MAC layer congestion issues have been investigated extensively. Numerous theoretical and simulation-based analyses show the impact of high density on latency and packet successful reception rate [6]. The study in [7] illustrates that high traffic of periodic broadcast messages may reduce the resource availability for the emergency messages. A Markov model is used in the article to analyze the dissemination delay of eventdriven emergency messages in the presence of low-priority periodic messages, but no specific algorithm is proposed to determine a good tradeoff to improve the performance and the mobility of vehicles is overlooked. [8] describes a DSRC 
congestion control scheme, based on maximizing channel throughput via distributed control of the safety message rate. Hafeez et al. in [6] propose a mobility model considering the threshold distance to avoid collision in different vehicle density indicated by a Poisson arrival queue. In [9], an adaptive rate control algorithm is proposed, which takes into account the prediction deviation of the motion of nearby vehicles. The broadcast will be deferred when the predicted positions satisfy the feasibility condition in consecutive slots. Few works have been done to consider the threshold distance in mobility models. Hafeez in [6] introduces the threshold distance in the initial distribution of vehicles, but the state of each vehicle is considered to be constant and independent. The majority of studies of congestion control in safety-related message focus on the performance improvement of delay and throughput. There have been no studies to explore the theoretical optimum of messages rate control on the premise of the reliability of security.

This paper proposes a mobility analytical model under highway scenarios, taking into consideration the probability of the change of motion in different density and velocity, which can reflect the mobility of vehicles more realistically. Then a novel information theory based rate control algorithm is introduced to obtain the theoretical optimum of messages rate to reduce redundant broadcast messages on the premise of the reliability of security under different scenarios. Entropy will be used in the rate control algorithm to measure the measure the uncertainty based on the probabilistic model we derived. Simulation results show that the proposed rate control algorithm can enhance the performance of DSRC system and make a good trade-off between the safety requirements and system performance in terms of delay and packet loss rate, aggravated by redundant broadcast messages which have little effects on safety applications. The rest of the paper is organized as follows. Section II describes the mobility analytical model. In Section III, the information theory based rate control algorithm is described along with the theoretical derivation based on the mobility model we proposed. Simulation results are given in Section IV to verify the performance and accuracy of the algorithm, and Section V presents the conclusion.

\section{Mobility Model}

Modeling the mobility of vehicles realistically is an essential and challenge task since the movement of the vehicle is determined by many factors such as the traffic density, the vehicles velocity and driving habits. Most related researches on vehicles mobility model neglect or simplify the changing status of vehicles caused by the traffic condition, driver's behavior. And the motion status is considered unchanged and independent throughout the entire process, which is unrealistic. In this paper, a new mobility model is proposed, considering the probability of status changes under different scenarios.

The proposed mobility model is based on a one-way oneline highway scenario, which can be extended to other more complicated scenarios. Since the transmission range is much larger than the wide of the road, the highway scenario is abstracted into a 1-D model. Vehicles will follow the direction of the road with a speed uniformly distributed between $V_{\min }$ and $V_{\max }$ [10]. The Poisson process is widely adopted as a sufficiently accurate assumption for modeling the vehicle arrival process in the highway scenario [3]. Vehicles in the highway scenario follow Poisson point process with density $\beta$ (in terms of vehicles per meter), and the vehicles spacing satisfies the exponential distribution. The PDF of vehicles spacing $S$ is given as

$$
f(s)=\beta e^{-\beta s}, s>0 .
$$

The safety distance $D_{s}$ is taken into account, which is determined by the current speed $V$ and the reaction time $T_{r}$. And the safety distance is given as

$$
D_{s}=V \times T_{r}
$$

To avoid collision caused by a sudden stop from the vehicle in front, the driver will decelerate when the distance from the vehicle ahead is detected smaller than the safety distance. At an arbitrary point in time, to find the probability that the vehicle will decelerate to keep a safety distance, the distributions of vehicle's density and speed are assumed constant is a certain period of time compared with the time of the communication process. Because the density and the speed are independent, the probability that the vehicle will decelerate at an arbitrary point in time can be described as

$$
\begin{aligned}
P_{d} & =\iint_{s<T_{r} \times V} f(s) \frac{1}{V_{\max }-V_{\min }} \mathrm{d} s \mathrm{~d} v \\
& =\frac{e^{-\beta T_{r}\left(e^{-V_{\min }}-e^{-V_{\max }}\right)}}{\beta T_{r}\left(V_{\max }-V_{\min }\right)} .
\end{aligned}
$$

The probability of deceleration in a poor traffic condition with dense traffic and high speed is derived in (3), which means that the vehicles dynamic states are frequently changed, consistent with the actual traffic situation. On the contrary, a vehicle prefers to remain the current state when the traffic condition is good. In the case that a vehicle won't decelerate, it will remain the current speed or accelerate with the probability of $1-P_{d}$. In this situation, the probability of accelerate is assumed to be proportional to $P_{d}$, which means that the probability of acceleration will increase with $P_{d}$ in a good traffic condition, and will decrease when $P_{d}$ is high. Based on the assumption and analysis above, the probability of acceleration can be written as

$$
P_{a}=P_{d}\left(1-P_{d}\right)
$$

And the probability that a vehicle remains the current state is

$$
P_{r}=1-P_{d}-P_{a} .
$$

From (3), (4) and (5), it's clear that vehicles are more likely to accelerate or decelerate in the high-density and high-speed scenario.

\section{AnAlytical Model And Information Theory Based Control Algorithm}

The Basic Safety Message (BSM) format [11] is utilized in the periodic broadcast messages of DSRC safety applications. The safety related messages are transmitted over 300-500 meters, with a $6 \mathrm{Mbps}$ data rate, and a $10 \mathrm{~Hz}$ message rate by default [12],[13]. The redundancy introduced by the fixed message rate scheme when the broadcast messages are excess to support safety applications will aggravate network traffic and increase the probability of packets loss and the packets 
delay, especially in dense and high-mobility conditions. The performance degradation can severely impact the time-critical safety applications.

In this section, an information theory based rate control algorithm is proposed based on the mobility model in Section II to analyze the optimum rate control scheme in substance. The entropy will be utilized to measure the uncertainty of vehicles position caused by the distribution of spacing and the mobility of vehicles based on the probabilistic model we derived.

The motion of a vehicle is divided in time slots of $\Delta t$, during which the state of the vehicle is assumed to remain. The state can be changed at the beginning of a new time slot with the probability derived in the mobility model. To simplify the analysis, the acceleration is assumed to be a fixed value. Then the probability distribution of acceleration of the vehicle in the $n_{i}$ slot based on (3), (4) and (5) is

$$
a_{i}=\left\{\begin{array}{rr}
a & P_{a} \\
0 & P_{r} \\
-a & P_{d}
\end{array}\right.
$$

The speed of the vehicle at the end of the $n_{i}$ slot is

$$
V_{i}=V_{i-1}+a_{i} \Delta t
$$

The distance the vehicle travels during the $n_{i}$ slot can be written as

$$
S_{i}=V_{i-1} \Delta t+\frac{1}{2} a_{i} \Delta t^{2} .
$$

The time a state packet is received from the target vehicle serves as a reference point $t_{0}$, and the speed $V_{0}$ and the position $X_{0}$ can be obtained from the received packet. Substituting the speed in the reference point, the speed of the vehicle at the end of the $n_{i}$ slot is given as

$$
\begin{aligned}
V_{i} & =V_{i-1}+a_{i} \Delta t \\
& =V_{0}+\sum_{k=1}^{i} a_{k} \Delta t .
\end{aligned}
$$

From the reference time $t_{0}$, the total distance in $N \Delta t$ is

$$
\begin{aligned}
S_{N \Delta t} & =\sum_{k=1}^{i} S_{i} \\
& =V_{i-1} \Delta t+\frac{1}{2} a_{i} \Delta t^{2} .
\end{aligned}
$$

Substituting (8) in (9), we have

$$
\begin{aligned}
S_{N \Delta t} & =\sum_{i=1}^{N}\left(V_{0}+\sum_{k=1}^{i-1} a_{k} \Delta t\right) \Delta t+\frac{1}{2} a_{i} \Delta t^{2} \\
& =N V_{0} \Delta t+\sum_{i=1}^{N}(N-i) a_{i} \Delta t^{2}+\frac{1}{2} a_{i} \Delta t^{2} \\
& =N V_{0} \Delta t+\sum_{i=1}^{N}\left(N-i+\frac{1}{2}\right) a_{i} \Delta t^{2}
\end{aligned}
$$

Assume that no state broadcast packet is received from the target vehicle since $t_{0}$, so the position prediction at $N \Delta t$ is based on the safety information obtained from the most recently received packet at $t_{0}$. The predicted position of the vehicle at $N \Delta t$ is

$$
\tilde{S}_{N \Delta t}=N V_{0} \Delta t
$$

Combining (10) and (11), the deviation of the prediction about the position of the vehicle at $N \Delta t$ is

$$
S_{N \Delta t}-\tilde{S}_{N \Delta t}=\sum_{i=1}^{N}\left(N-i+\frac{1}{2}\right) a_{i} \Delta t^{2} .
$$

Based on the assumption that the motion of every slot is independent, the deviation of prediction is composed of $N$ independent variables as

$$
\begin{aligned}
& S_{N \Delta t}-\tilde{S}_{N \Delta t}=\sum_{i=1}^{N} X_{i} \\
& X_{i}=\left(N-i+\frac{1}{2}\right) a_{i} \Delta t^{2} .
\end{aligned}
$$

According to the Liapunov central limit theorem, the deviation of prediction is approximate normal distribution.

$$
S_{N \Delta t}-\tilde{S}_{N \Delta t}=\sum_{i=1}^{N} X_{i} \sim N\left(\mu, \sigma^{2}\right) .
$$

where $\mu$ and $\sigma^{2}$ are summations of the mean and variance of each independent variable $X_{i}$. According to the probability distribution of the acceleration in (6), $\mu$ and $\sigma^{2}$ can be expressed as

$$
\begin{aligned}
\mu & =\sum_{i=1}^{N} E X_{i} \\
& =\sum_{i=1}^{N}\left(N-i+\frac{1}{2}\right) a_{i} \Delta t^{2}\left(P_{a}-P_{d}\right) \\
& =\frac{N^{2} a_{i} \Delta t^{2}\left(P_{a}-P_{d}\right)}{2}
\end{aligned}
$$

$$
\begin{aligned}
\sigma^{2} & =\sum_{i=1}^{N} D X_{i} \\
& =\sum_{i=1}^{N} P_{a}\left[\left(N-i+\frac{1}{2}\right) a_{i} \Delta t^{2}\right]^{2}+P_{d}\left[\left(N-i+\frac{1}{2}\right) a_{i} \Delta t^{2}\right]^{2} \\
& =\frac{\left(P_{a}+P_{d}\right) a^{2} \Delta t^{4}\left(4 N^{3}-N\right)}{12} .
\end{aligned}
$$

From (16), (17) and (18), it is clear that the accuracy of prediction decreases with the number of time slots. To guarantee the performance of safety applications in vehicles positioning and collision warning, the threshold $E_{t h}$ of the deviation of position prediction is set as $0.5 \mathrm{~m}$ based on the analysis in [14]. Therefore, the probability that the positioning deviation of the target vehicle is less than the threshold is given by

$$
\begin{aligned}
& P\left\{\left|S_{N \Delta t}-\tilde{S}_{N \Delta t}\right| \leq E_{t h}\right\} \\
& =\Phi\left(\frac{E_{t h}-\mu}{\sigma}\right)-\Phi\left(\frac{-E_{t h}-\mu}{\sigma}\right) .
\end{aligned}
$$


In this paper, we consider that ensuring the positioning deviation less than the threshold with $95 \%$ probability is acceptable. Based on the analysis above, we get

$$
\begin{gathered}
\Phi\left(\frac{E_{t h}-\mu}{\sigma}\right) \approx \Phi(2) . \\
\sigma_{t h} \approx \frac{E_{t h}-\mu_{t h}}{2} .
\end{gathered}
$$

To measure the uncertainty of the positioning deviation, the entropy the uncertainty $Y$ at $N \Delta t$ is

$$
H(Y)=\ln \left(\sigma_{N} \sqrt{2 \pi e}\right)
$$

where $\sigma_{N}$ is the standard deviation of the distribution at $N \Delta t$

From (20) and (21), the threshold of the entropy at $N \Delta t$ is $\ln \left(\sigma_{t h} \sqrt{2 \pi e}\right)$. Assume that a state packet is received at $N^{\prime} \Delta t$ during $t_{0}$ to $N \Delta t$, the conditional entropy is

$$
H(Y \mid X)=\ln \left(\sigma_{Y \mid X} \sqrt{2 \pi e}\right)
$$

where $\sigma_{Y \mid X}$ is the standard deviation of the distribution at $N \Delta t$ when a state packet is received at $N^{\prime} \Delta t$.

To satisfy the threshold of the entropy, we get the inequality

$$
\begin{aligned}
& \sigma_{Y \mid X} \leq \sigma_{t h} . \\
& \sigma_{Y \mid X}^{2}= \sum_{i=1}^{N-N^{\prime}} D X_{i} \\
&= \frac{\left(P_{a}+P_{d}\right) a^{2} \Delta t^{4}\left[4\left(N-N^{\prime}\right)^{3}-\left(N-N^{\prime}\right)\right]}{12} .
\end{aligned}
$$

Substituting (25) in (24), the maximum transmission interval $\left(N-N^{\prime}\right) \Delta t$ under the given density and velocity distribution can be derived.

\section{Model Validation And Simulation}

In this section, the optimal periods under various conditions and the accuracy of scheme are shown according to the information theory based model we proposed. The well-known simulator NS3 [15] (version 3.22) is used to analyze the performance of the rate control algorithm in Section III. Fig. 1 shows the optimal period of the broadcast messages which can be calculated from (24) and (25) under different vehicle density and velocity distributions. From the numerical results in Fig. 1 , it can be observed that the value of the broadcast period is significantly different under various conditions. The fixed rate control scheme can't meet the changing security requirements and redundancy will be introduced in some circumstances.

Fig. 2 shows the accuracy of the position prediction through the periodic broadcast messages with the rate control scheme we proposed. The motion of the vehicle is based on the mobility model in Section II. The vehicles spacing satisfy the exponential distribution with $\beta=0.05$, and the speed ranges from $80-120 \mathrm{~km} / \mathrm{h}$, typical for the highway scenario. The Nakagami propagation model is used in simulations, which is considered best for vehicular environment in many research [16], [17]. All the vehicles will broadcast state messages based on the rate control algorithm we proposed, the emergency messages will be sent from a chosen vehicle, and forwarded by the vehicles behind. To analyze the performance of the

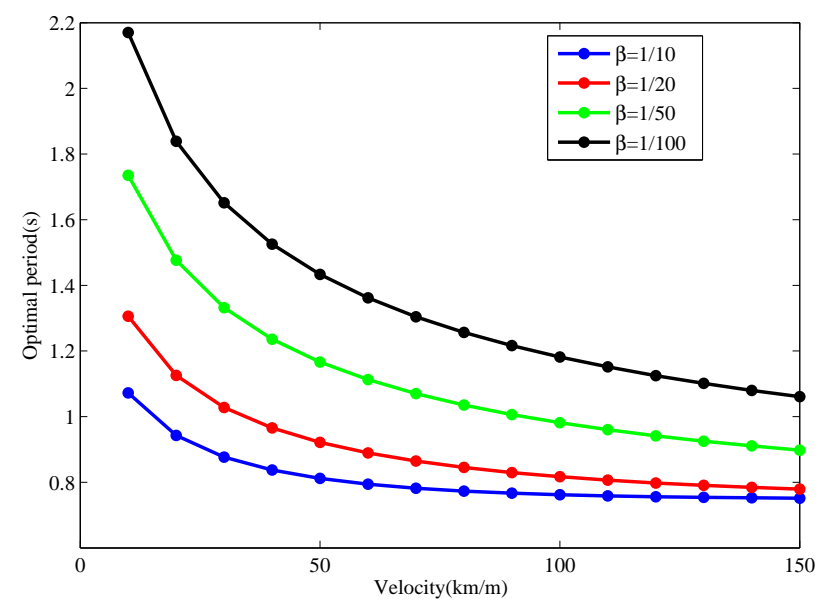

Fig. 1. Optimal periods of the broadcast messages under different vehicle density and velocity distributions

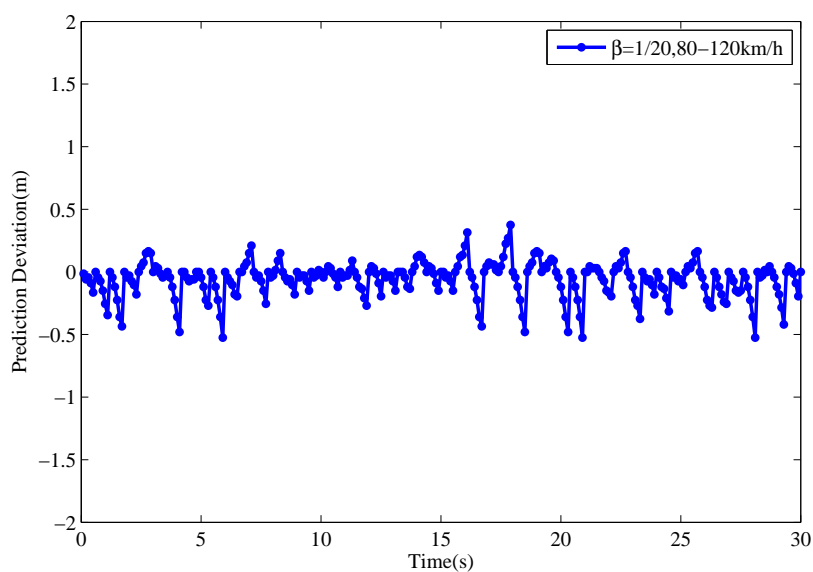

Fig. 2. Prediction deviation with $\beta=1 / 20$, speed ranges from $80-120 \mathrm{~km} / \mathrm{h}$

information theory based rate control algorithm, two metrics are defined as follow: 1) the packet loss rate, the percentage of the total number of vehicles in the transmission range that fail to receive the broadcast messages. 2) the average delay to broadcast state messages. The simulation parameters are summarized in Table 1.

TABLE I

VALUE OF PARAMETERS USED IN SIMULATION

\begin{tabular}{|c|c|}
\hline Parameter & Value \\
\hline Length of highway segment & $2000 \mathrm{~m}$ \\
\hline Vehicle's speed & $80-120 \mathrm{~km} / \mathrm{h}$ \\
\hline Vehicle's density & $0.01-0.1 \mathrm{vehicle} / \mathrm{m}$ \\
\hline Frequency & $5.9 \mathrm{GHz}$ \\
\hline Packet size & $100 \mathrm{Bytes}$ \\
\hline Transmission power & $50 \mathrm{~mW}$ \\
\hline Received power threshold & $3.162 \mathrm{e}-13 \mathrm{~W}$ \\
\hline Noise floor & $1.26 \mathrm{e}-14 \mathrm{~W}$ \\
\hline DIFS & $64 \mu \mathrm{s}$ \\
\hline Antennas gain & 1 \\
\hline Reaction time & $1 \mathrm{~s}$ \\
\hline$\Delta \mathrm{t}$ & $100 \mathrm{~ms}$ \\
\hline
\end{tabular}




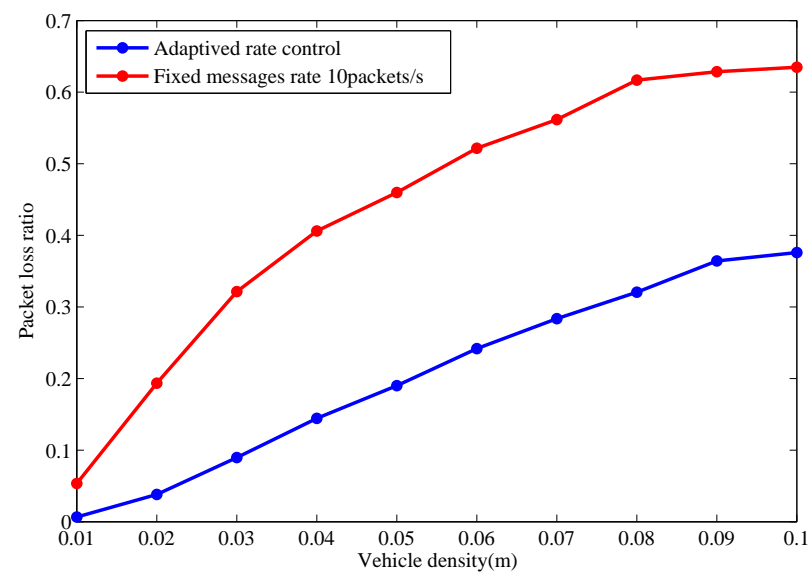

Fig. 3. Packet loss rate versus vehicle's density

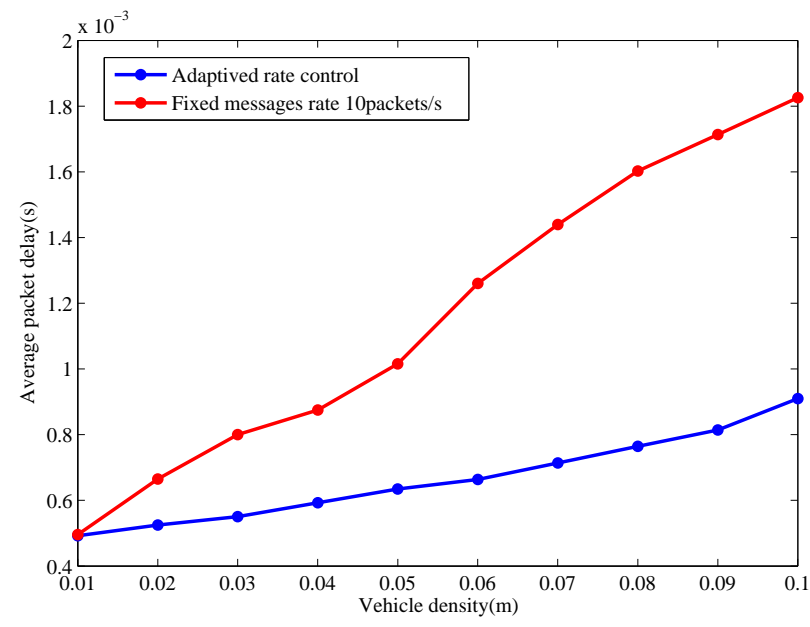

Fig. 4. Packet delay versus vehicle's density

Fig. 3 and Fig .4 show, respectively, the packet loss rate the packet delay versus vehicle density ranged from 0.01 to 0.1 vehicles $/ \mathrm{m}$ in (1) with and without the entropy-based rate control algorithm. As is shown in Fig. 3, the packets loss caused by collisions from neighboring vehicles and hidden terminals will become serious with the increase of vehicle density, which will leads to undesirable performance, particularly in dense vehicular environment. At the same time, vehicles will take longer time to access the channel to broadcast the state messages, and the packet delay increases with the increasing of vehicles density. The high packet loss rate and packet delay will be a disaster to safety applications. Adapting the entropy-based rate control algorithm, the packet loss rate and packet delay are improved greatly, as is shown in the figure. It is obvious that the entropy-based rate control scheme outperforms fixed-rate and other rate control schemes not only for the improvements of network performance, but also the consideration to the security requirements.

\section{CONCLUSIONS}

In this paper, a new mobility model has been presented in which the change of motion under various vehicle densities and speeds is considered. Entropy is used to describe the uncertainty of the vehicle position caused by the change of motions, and an information theory based rate control algorithm is proposed to derive the optimum broadcast period. Adapting the rate control algorithm, vehicles are able to adjust the broadcast period of states messages to improve the performance of safety applications. Simulation results in NS3 show that the proposed rate control algorithm can significantly reduce redundancy, thus reducing the packet delay and packet loss rate, and the accuracy of vehicle positioning is guaranteed at the same time. Compared with existing researches, the system performance and safety requirements are both taken in to considered, and a good trade-off is achieved.

\section{ACKNOWLEDGMENT}

We would like to thank the experts from Qualcomm for their valuable comments.

\section{REFERENCES}

[1] J. B. Kenney, "Dedicated short-range communications (dsrc) standards in the united states," Proceedings of the IEEE, vol. 99, no. 7 , pp. 1162-1182, 2011.

[2] U. F. C. Commission et al., "R\&o fcc 03-324,," Dedicated Short Range Communications Report and Order, 2003.

[3] Y. Yao, L. Rao, and X. Liu, "Performance and reliability analysis of ieee $802.11 \mathrm{p}$ safety communication in a highway environment," Vehicular Technology, IEEE Transactions on, vol. 62, no. 9, pp. 4198-4212, 2013.

[4] S. Biswas, R. Tatchikou, and F. Dion, "Vehicle-to-vehicle wireless communication protocols for enhancing highway traffic safety," Communications Magazine, IEEE, vol. 44, no. 1, pp. 74-82, 2006.

[5] X. Wu, S. Subramanian, R. Guha, R. G. White, J. Li, K. W. Lu, A. Bucceri, and T. Zhang, "Vehicular communications using dsrc: challenges, enhancements, and evolution," Selected Areas in Communications, IEEE Journal on, vol. 31, no. 9, pp. 399408, 2013.

[6] K. A. Hafeez, L. Zhao, B. Ma, and J. W. Mark, "Performance analysis and enhancement of the dsrc for vanet's safety applications," Vehicular Technology, IEEE Transactions on, vol. 62, no. 7, pp. 3069-3083, 2013.

[7] M. Khabazian, S. Aïssa, and M. Mehmet-Ali, "Performance modeling of safety messages broadcast in vehicular ad hoc networks," Intelligent Transportation Systems, IEEE Transactions on, vol. 14, no. 1, pp. 380-387, 2013.

[8] G. Bansal and J. B. Kenney, "Controlling congestion in safetymessage transmissions: A philosophy for vehicular dsrc systems," Vehicular Technology Magazine, IEEE, vol. 8, no. 4, pp. 20-26, 2013.

[9] H.-H. Nguyen and H.-Y. Jeong, "Adaptive beacon rate control algorithm for vehicular ad-hoc networks," in Ubiquitous and Future Networks (ICUFN), 2013 Fifth International Conference on. IEEE, 2013, pp. 652-653.

[10] K. A. Hafeez, L. Zhao, Z. Liao, and B. Ma, "Impact of mobility on vanets' safety applications," in Global Telecommunications Conference (GLOBECOM 2010), 2010 IEEE. IEEE, 2010, pp. $1-5$.

[11] D. Committee et al., "Dedicated short range communications (dsrc) message set dictionary," 2009.

[12] J. B. Kenney, G. Bansal, and C. E. Rohrs, "Limeric: a linear message rate control algorithm for vehicular dsrc systems," in Proceedings of the Eighth ACM international workshop on Vehicular inter-networking. ACM, 2011, pp. 21-30.

[13] C. V. S. C. Consortium et al., "Vehicle safety communications project: task 3 final report: identify intelligent vehicle safety 
applications enabled by dsrc," National Highway Traffic Safety Administration, US Department of Transportation, Washington $D C, 2005$

[14] S. E. Shladover and S.-K. Tan, "Analysis of vehicle positioning accuracy requirements for communication-based cooperative collision warning," Journal of Intelligent Transportation Systems, vol. 10, no. 3, pp. 131-140, 2006.

[15] "Ns-3 network simulator," http://www.nsnam.org, [Online;Available:].

[16] F. Schmidt-Eisenlohr, M. Torrent-Moreno, J. Mittag, and H. Hartenstein, "Simulation platform for inter-vehicle com- munications and analysis of periodic information exchange," in Wireless on Demand Network Systems and Services, 2007. WONS'07. Fourth Annual Conference on. IEEE, 2007, pp. $50-58$.

[17] V. Taliwal, D. Jiang, H. Mangold, C. Chen, and R. Sengupta, "Empirical determination of channel characteristics for dsrc vehicle-to-vehicle communication," in Proceedings of the 1st ACM international workshop on Vehicular ad hoc networks. ACM, 2004, pp. 88-88. 